\section{Incidence of hypertension in a high- risk workgroup (Police officers) - Observational study}

\author{
Guillermo Padrón Arredondo*
}

General Surgeon, Surgery Service of Hospital General Playa del Carmen, Constituyentes Avenue with Avenue 135 Ejido Suburb, Playa del Carmen, Quintana Roo. Mexico

\section{Summary}

Introduction: Hypertension is a silent pathology in a way that affects all four spheres to be considered as such; magnitude, transcendence vulnerability, and feasibility. The World Health Organization estimates that $45 \%$ of deaths from heart disease and $51 \%$ of deaths from stroke globally are caused by hypertension.

Material and method: A longitudinal, descriptive and quantitative observational study was carried out on the personnel of high-risk public service providers.

Results: The total population sampled was 550 people where it was possible to determine the sex where the disease predominates, since $92 \%$ of the hypertensive population belong to the male sex, while $8 \%$ of the female population. $57 \%$ of the total population were classified as normotensive, while $21 \%$ were classified as High Normal, Grade I Hypertension, and Grade II Hypertension.

Discussion: AHT is the result of a series of interactions between endogenous and exogenous factors in an organism that tries to adapt to the increase of the cardiac output and the peripheral resistance of the blood vessels, which is manifested by the increase in blood pressure figures. Physical activity has been shown to have a lower risk of hypertension compared to sedentary individuals. The daily stress these workers face predisposes them to suffer their manifestations as headache, muscle pain, fatigue, digestive disorders and constant elevations of blood pressure.

\section{More Information}

*Address for Correspondence: Guillermo Padrón Arredondo, General Surgeon, Surgery Service of Hospital General Playa del Carmen, Constituyentes Avenue with Avenue 135 Ejido Suburb, Playa del Carmen, Quintana Roo. Mexico, Tel: 01-984-206-1691;

Email: hospitalplay@hotmail.com gpadronarredondo@hotmail.com

Submitted: 30 October 2019

Approved: 07 November 2019

Published: 08 November 2019

How to cite this article: Arredondo GP. Incidence of hypertension in a high-risk workgroup (Police officers) - Observational study. Ann Clin Hypertens. 2019; 3: 052-058.

DOI: dx.doi.org/10.29328/journal.ach.1001020

Copyright: (c) 2019 Arredondo GP. This is an open access article distributed under the Creative Commons Attribution License, which permits unrestricted use, distribution, and reproduction in any medium, provided the original work is properly cited.

Keywords: Epidemiology; Hypertension; Prevalence; Risk-factors; Psycho-social stressors; Police officers; Outdoor activity

Check for updates

\section{Introduction}

Hypertension (HT) is a silent pathology that affects the four spheres to be considered as such; magnitude, transcendence, vulnerability, and feasibility. The estimate of the World Health Organization (WHO) is that $45 \%$ of deaths from heart disease and $51 \%$ of deaths from stroke worldwide are caused by hypertension. Added to according to data from the National Health and Nutrition Survey (ENSANUT 2012) of every 1000 Mexicans 315 suffer from HTA and half of them do not know it, that is, it affects $31.5 \%$ of Mexicans and is considered one of the most prevalent high worldwide $[1,2]$.

Being a disease that is preventable through a healthy diet and exercise, in short a disease that can be prevented by adopting healthy lifestyles and once detected can be controlled by pharmacological and non-pharmacological treatment, therefore, it is of utmost importance take measures for its detection and timely follow-up and avoid the long-term consequences that the disease brings, which are the real public health problem.

\section{Material and Method}

Across-sectional, descriptive and quantitative experimental study was carried out for the personnel of high-risk public service providers in the Municipality of Solidaridad Quintana Roo and the takings were made in a period of 3 consecutive weeks.

The population studied were men and women from 20 to 60 years of age who had their blood pressure taken with prior authorization with the following inclusion criteria: not having used tobacco, coffee or any other stimulant (e.g. chocolate, alcohol, tea, drugs) at least 60 minutes prior to the appointment, not having performed physical activity and not having sexual intercourse at least 4 hours prior to the appointment; At the time of their arrival they were asked to rest for 5 minutes and then the blood pressure was taken (TA); they will be placed in a sitting position with the left arm at the level of the heart, feet on the floor (without crossing the legs) and the back glued to the back of the chair. Early detection 
was based on taking blood pressure utilizing a calibrated sphygmomanometer.

The population that recorded high levels or outside the normal parameters during the first TA session would be given a new appointment to take the TA again, given that in order to establish a suspicion of HT, three random shots would be taken, if in the second shot will result in newly elevated figures, they will be cited again to confirm the diagnosis. Exclusion criteria: the population that had normal figures during the first TA session. Elimination criteria: Personnel that did not comply with the three TA taps. The TA measurement will be carried out with a double-hose aneroid sphygmomanometer. The general objective was to demonstrate the incidence of hypertension in this group of service providers to propose alternative solutions taking as a reference frame the result of the data obtained during the study.

\section{Results}

The total population sampled was 550 people (100\%) where it was possible to determine the sex where the pathology prevails more than $92 \%$ of the hypertensive population belonging to the male sex, while $8 \%$ of the female sex (Figure 1).

$57 \%(\mathrm{n}=311$ people) of the total population resulted in the category that classifies them as normotensive so that their BP figures were in a range of 120-129 $\mathrm{mmHg}$ systolic and 80-84 mmHg diastolic. While $21 \%(n=113)$ were classified in the category of Normal high, Hypertension grade I, and Hypertension grade II (Figure 2). 23\% $(\mathrm{n}=126)$ had an Undetermined result for not meeting the taking of 3 random samples.

Of the 113 people with "normal high" and some degree of "HTA" were classified into the following categories: 54 items correspond to "normal high" since they maintained a range of 130-139 mmHg systolic and 85-89 mmHg diastolic; 49 items were classified in "Hypertension grade I" since they maintained BP figures in a range of 140-159 mmHg systolic and $90-99 \mathrm{mmHg}$ diastolic and finally 10 elements were classified in the category of "Hypertension grade II" since they maintained figures within the range of $160-179 \mathrm{mmHg}$ systolic and 100-109 mmHg diastolic (Figure 3). 18 (16\%) of these people already had a previous diagnosis of HTN. That is, $21 \%$ of the total population $(n=550)$ were found with some degree of HBP and $9 \%(n=54)$ of the total population $(n=$ 550) have developed risk factors (high normal) that although not they enter the classification of HTA and in the future they could develop HTA in any of their degrees.

\section{Discussion}

HT is a pathology that can be fatal and is irreversible because its signs and symptoms remain dormant for a long time; its primary manifestations are uncertain and may go unnoticed, so it is considered a "silent" disease. Many risk factors have been attributed to a person can present or develop hypertension
Percentage of patients with hypertension.

Normal

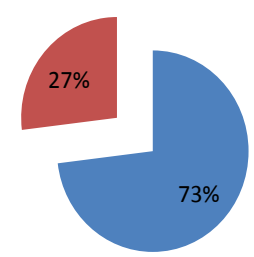

Figure 1: Percentage $(\%)$ of 113 men and women with data on arterial hypertension in a population at risk.

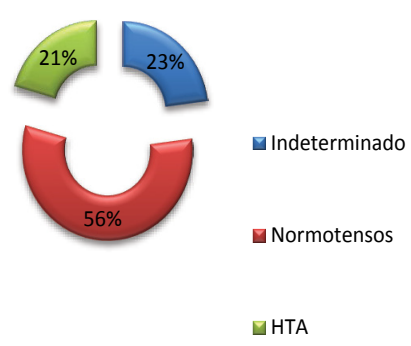

Figure 2: Percentage (\%) of Arterial Blood Pressure in 550 people in a risk group.

normal alta

- Hipertension Grado I

Hipertension Grado II

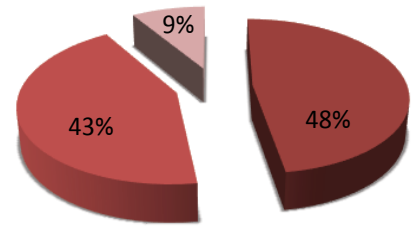

Figure 3: Classification according to the state of HTA in 113 police officers of risk.

during his life, most are factors that can be modified because they are those that are directly related to lifestyle for example; sedentary lifestyle, high salt intake in the diet, lack of physical activity, smoking, obesity. And there are non-modifiable factors, for example; race, sex, family history, and age. The development of secondary diseases such as heart failure, myocardial infarction or brain supposes a high economic cost for the Health Sector due to the need for high-cost medications, hospital stay, rehabilitation, surgeries, etc. For a disease that can be preventable and can be controlled, and because the prevalence of HBP is currently focused on the population of young adults and the elderly, a public health alert has been activated in which measures of prevention of risk behaviors and control where the early detection of the pathology gives the guide to reduce the morbidity and mortality rates because of the complications that long-term hypertension brings with it.

The Clinical Practice Guideline for the Diagnosis and Treatment of HBP in the first level of care (2014) has given 
as frame of reference stratification in which according to the blood pressure that is taken to the patient, the figures will be classified and defined. Obtained, these references were taken from the European Heart Journal [3] (Table 1).

On the other hand, it was determined that pharmacological treatment was received in greater proportion by the population older than 70 years compared to those aged 20 to 45 years and when comparing the results with the previous ENSANUT carried out in 2000 and 2006 with the one carried out in 2012 and subsequently contrasted it was found that the trends in the Mexican population they have followed a steady path since it has not undergone changes: $32 \%$ in 2012; $30 \%$ in 2000 , and $32 \%$ in 2016 where, despite everything, a prevalence of HBP has been maintained in the male and female sex at least in the last six years: $32 \%$ 2006; 32\% 2012, in males and $31 \%$ in 2006; 31\% 2012, in females [4].

HT is the result of a series of interactions between endogenous and exogenous factors in an organism that tries to adapt to the increase in cardiac output and the peripheral resistance of the blood vessels, which is manifested by the elevation in the high blood pressure (HBP).

As mentioned, there are risk factors and predisposing factors for a person to develop hypertension, so we have endogenous factors that cannot be modified as they are; the black race, the male sex, family predisposition and the hormonal state, and there are also endogenous factors that are related to the lifestyle of people such as high salt intake in the diet, sedentary lifestyle, unhealthy diet, smoking, alcohol consumption, and stress. The importance in public health is that because HBP remains asymptomatic for a long period it is not detected in time and hypertensive patients, "unknowingly", are predisposed to suffer a series of medical complications that are nothing other than the result long term that brings this silent disease as: acute myocardial infarction, cerebrovascular events, renal failure, dyslipidemia, and glucose intolerance, which have repercussions on a personal level since it decreases the quality of life and can cause premature death if we compare with the life expectancy currently estimated, as well as economic since they need more medicines and of greater cost, and in public health since it is estimated that if not acted on time, cardiovascular diseases, stroke, among other no communicable diseases will go increasing, causing a high cost in terms of health, such as special surgeries, specific

\begin{tabular}{|c|c|c|}
\hline Table 1: Values of TA in the evaluation of Systemic Arterial Hypertension. & Diastolic $(\mathbf{m m H g})$ \\
\hline Category & $<120$ & $<80$ \\
\hline Óptima & $120-129$ & $80-84$ \\
\hline Normal & $130-139$ & $85-89$ \\
\hline Normal high & $140-159$ & $90-99$ \\
\hline Hypertension, grade 1 & $160-179$ & $100-109$ \\
\hline Hypertension, grade 2 & $>=180$ & $>=110$ \\
\hline Hypertension, grade 3 & $>=140$ & $>90$ \\
\hline Isolated systolic hypertension & & S \\
\hline
\end{tabular}

Source: Diagnosis and Treatment of Arterial Hypertension in the First Level of Care, 2013. medications with high cost and hospital stay of those affected a situation that can be prevented by modifying and making people aware so that they modify their lifestyles.

Physical activity has shown a lower risk of hypertension compared to sedentary people. In studies with physically active people, they had 10 times fewer chances of hypertension than those who did not exercise.

It has been reported that the accumulation of physical activity decreases BP in both hypertensive and prehypertensive people but not in normotensive patients, however, physical activity generally helps to decrease other risk factors associated with hypertension such as for overweight and obesity [5].

Hypertension, hypercholesterolemia and smoking constitute the three main risk factors for the development of atherosclerosis. Therefore, hypertension is the first factor causing atherothrombotic cerebral strokes; the second factor causing myocardial infarction, and the third factor causing atherosclerotic obliterans disease of the lower extremities. These data give an idea of the role played by hypertension in the high morbidity and mortality associated with atherosclerosis [6].

Arterial hypertension (HBP) has different etiologies and yet none is definitive since as mentioned above several factors intervene, currently with 95\% prevalence the type of HBP that affects the population is essential or idiopathic.

Since this type of HBP is not the result of a disease, a series of risk factors that predispose people to manifest the disease have been attributed as the main factor. Some risk factors for hypertension are: people who have a direct family member with HBP, as well as elderly people who have direct family members with HBP are predisposed, this makes reference to a direct genetic predisposition that although it is not clear, it is sufficient to consider it a risk factor, people who have metabolic disorders such as obesity and insulin resistance as in the case of Diabetes mellitus type 2 also have predisposition since not being able to use glucose properly it must be eliminated from the body which will stimulate the function sympathetic looking to increase the metabolic rate and eliminate calories and later increase the cardiac work in blood vessels and the kidney can manifest the pathology, these can be considered factors linked to the individual or endogenous.

On the other hand, we find exogenous factors or related to the lifestyle that is directly related to the behaviors of people predisposing them to the pathology; the high consumption of sodium in the diet has been related to the increase in blood volume as well as in the function of the kidney and its adrenergic functions, people who consume alcoholic beverages regardless of ethnicity or sex have high systolic figures, lack of potassium consumption in the diet is an important factor since it has a direct diuretic effect favoring the elimination of salt by effects on the renin-angiotensin-aldosterone system 
that regulates the $\mathrm{BP}$, the female hormones present in oral contraceptives of women as estrogen and progesterone are responsible for $5 \%$ of the HBP that is reversed with the suspension of the contraceptive.

Stress in people in a predisposing factor because during situations of malaise and crisis the AT tends to rise which could cause hypertrophy in the vascular smooth muscle. Certainly we can say that we all have or could develop stress throughout life in different circumstances and situations and since we talk about stress it is important to define the concept: WHO defines stress as "the set of physiological reactions that prepare the body for the action "it is important to mention that there are studies that have revealed which are the jobs or more stressful professions; The importance of these studies is that the police profession enters into this classification because they are working in an area where they face conflicts and risks inherent to their daily work, in addition to the fact that in the area where they operate they deal directly with the citizenship, added to this can be added factors such as; equipment problems, extended hours of work shift, and problems related to the community that, to name a few, would be apathy towards the public, negative image of the police, poor support at work, etc. The daily stress faced by these workers predisposes them to suffer its manifestations as headache, muscle pain, fatigue, digestive disorders and constant elevations of blood pressure.

The Americas region has a prevalence of hypertension of $35 \%$, in the same way, research shows that the prevalence of hypertension is higher in low and middle-income countries with $45 \%$, it should be mentioned that countries that fall into the category aforementioned are more likely to have weak health systems with a high population causing hypertensive people to remain without a diagnosis, treatment or control. In contrast, in high-income countries, the prevalence is $35 \%$ [2].

The prevalence of HT in Mexico is $32 \%$ of which up to $47 \%$ did not know about the disease. Adults older than 60 years have a higher prevalence of HBP than the group of 20 to 29 years of age and the established diagnosis of hypertension was higher in those who were older than 60 years. Likewise, the prevalence varied depending on the region, where the northern regions of the country were more prevalent than in the southern regions, and in urban areas, it was higher than in the rural regions.

In a study on prevalence between prehypertension and arterial hypertension in police officers between 25 and 39 years of age, it was found between 38 and 35\% concluding that in this group of people it is comparatively higher than in the general population. The decrease in alcohol consumption, fruit consumption as well as supported by changes in lifestyle can help in the prevention and control of high blood pressure [7].

Diabetes mellitus and hypertension are common diseases in adults and in a study where they assessed the prevalence of cardiovascular risk factors, diabetes mellitus, hypertension, smoking, alcoholism, and obesity in the police, it was found that these factors are present in the staff police [8].

Campos-Nonato, et al. [9] consider that in order to increase the percentage of hypertensive adults with timely diagnosis, who have blood pressure levels under control and with fewer complications, it is important to 1) implement programs that allow early diagnosis and care timely of adults who are at risk of developing hypertension, with the goal of improving the figures identified in the ENSANUT 2012; 2) establish the use of clinical protocols in all personnel responsible for caring for patients with hypertension, to prescribe the best and most upto-date treatment. With this, you should increase the number of hypertensive adults with blood pressure levels under control; 3) to ensure that health promotion and prevention and treatment programs for HTA are designed with the adoption of healthy lifestyles as the main axis, which include: discouraging the initiation of smoking and cessation among addicts, maintaining a diet with low content of saturated fats, trans fats, cholesterol, sodium and alcohol, but with high consumption of vegetables, whole-grain cereals and low-fat dairy; In addition, promote the constant practice of moderatevigorous physical activity of at least 150 minutes per week; 4) promote the reformulation of industrialized foods to improve their profile (looking for low sodium, total fat, saturated and trans fat, but with a high fiber content) $[3,9]$.

Despite the fact that measures have been taken at the national level for timely diagnosis and education to reduce risk factors, no impact on public health has been achieved since it was found that people with pharmacological treatment to control HBP maintain high levels of TA which indicates apathy towards adherence to treatment, at the pace at which the current situation of the country develops according to projections to the year 2025 the non-communicable diseases the hypertension will be one of the first causes of mortality due to an entire epidemiological transition that has been emerging since the 80 s when people died from infectious diseases and now coupled with a life expectancy that for the Mexican population is 78 years for women and 73 years for men will have increased the population of senior citizens who will not have a normal or healthy aging but on the contrary they will reach the third age with a series of pathologies that will cost millions of pesos to maintain.

HTA, like other diseases, has treatment in which the objective is to reduce the cardiovascular comorbidity that it brings, for this reason, two types of treatment has been determined to control the pathology: pharmacological and non-pharmacological treatment which is based mostly on modifications in lifestyle and eating habits.

In the Municipality of Solidaridad, Quintana Roo. Mexico, most of the public service providers spend their work shifts 
with enough work that does not allow them to have a healthy diet because with the 30 minutes they are designated, they do not allow enough time to eat properly or carry a healthy diet, in addition to the time they dedicate to work does not allow them to carry out any physical activity coupled with a lack of culture or ignorance of the importance of health to have a healthy diet and exercise at least 30 minutes a day.

Half of the population of the public security and municipal transit police officers, due to an insane diet and sedentary lifestyle, are prehypertensive or hypertensive and do not know it. Non-pharmacological treatment refers to improving physical activity and diet therefore in patients with HBP it will be recommended; the salt intake should be 5-6 mg per day however it is these patients consumption will be reduced to less than 2 grams per day of sodium (patients under 50 years $1500 \mathrm{mg}, 51-70$ years $1300 \mathrm{mg}$ and older than 70 years 1200 $\mathrm{mg}$ ) there is evidence that they can reduce up to $3.47 \mathrm{mmHg}$, hypertensive patients should have a body mass index (BMI) of 25 , achieving a reduction of $4.4 \mathrm{mmHg}$ systolic and $3.6 \mathrm{mmHg}$ diastolic pressure.

The incorporation to a diet "Dietary Approaches to Stop Hypertension" (DASH) is based on the consumption of a diet rich in fruits and vegetables, low in saturated and total fats, this diet is rich in magnesium, potassium, calcium, proteins and fiber managing to reduce up to 8-14 $\mathrm{mmHg}$, which consists of a reduction in the consumption of fats, red meat, sweets and sugary drinks, increasing the consumption of fruits and vegetables (300-400 g per day), low-fat dairy, fiber dietary and soluble, whole grains and proteins of vegetable origin, poultry meat, fish (twice a week) and nuts.

The consumption of coffee and alcohol will be reduced to 20-30 $\mathrm{g}$ in men and 10-20 $\mathrm{g}$ in women, achieving a reduction of 2-4 mmHg of SBP systolic blood pressure and diastolic blood pressure. The performance of aerobic physical activity is one of the main axes of this treatment, reducing the SBP and DBP by 4-9 $\mathrm{mmHg}$, the recommendation will be: 30 minutes of 5 to 7 days per week and 30-60 minutes of $4-7$ days a week. Promote the abandonment of tobacco and of course the incorporation into support groups for the control of modifiable risk factors. It should include in public health the implementation of policies in the food industry seeking to reduce the consumption of salt in the diet of the population, since by this means up to $80 \%$ of sodium is consumed and it will be recommended to choose cereals for breakfast with low sodium content, avoid the consumption of cured products (ham, bacon) canned foods in saltwater and condiments, limit the consumption of soy, teriyaki sauce, English sauce, ketchup and mustard, reduce the consumption of instant rice and precooked meals .

Pharmacological treatment will be chosen according to the degree of severity of the HTA since for its initiation as a therapeutic regimen monotherapy or more than one drug are used, the most used drugs or the first-line drugs are: thiazide-type diuretics act by decreasing renal absorption of sodium, reducing the volume vascular and propitiating salt and water elimination; angiotensin II converting enzyme (ACE) inhibitors act by inhibiting angiotensin I from becoming angiotensin II, reducing its effects such as vasoconstriction, aldosterone concentration, renal blood flow, and glomerular filtration rate; Angiotensin II receptor antagonists (ARA II) act by blocking angiotensin II receptors and consequently their effects; calcium antagonists inhibit calcium from entering the smooth muscle and cardiac muscle by decreasing muscle tone and therefore the TA; and the beta-blockers decrease the heart rate and the cardiac minute volume on the other hand also diminish the effects of the renin-angiotensin-aldosterone system on the TA. For the use of drugs, it is recommended to start with low doses that are subsequently adjusted according to the patient's response, if it is necessary to use more drugs simultaneously, the use of thiazides -IECA or IRA II- calciumantagonists is recommended.

Pharmacological treatment should be started when the patient maintains SBP levels $\geq 140 \mathrm{mmHg}$ and / or DBP $\geq 90$ $\mathrm{mmHg}$, two weeks later or simultaneously with changes in lifestyle. It has been shown that leading a sedentary lifestyle is a risk factor to develop hypertension. This can be added to an insane lifestyle that is, not performing the exercise and other behaviors. In the Municipality of Solidaridad, it can be observed that most of the time the Public Safety and Municipal Transit workers carry out their work inside their vehicles from 12 to 24 hours of work per day, it is also possible that due to a short period of the time they have to eat is the need to eat unhealthy and high-calorie food such as fried foods or fast food.

In a study by Shiozaki, et al. [10], they found that the development of myocardial ischemia was significantly associated with known risk factors, especially with hypertension, dyslipidemia and glucose intolerance. The increase with age of the prevalence of myocardial ischemia and the increase in metabolic syndrome was greater in police officers than in the office employees of the same corporation. Irregular working conditions in shifts, prolonged working hours and inappropriate lifestyles can influence the prevalence of cardiac ischemia in police officers.

On the other hand, it has also been found that in the security forces obesity is more frequent than in civilians while diabetes mellitus is lower. Also, these workers are exposed to intense and sudden physical exertion, acute and chronic psychological stress, shift work and noise. Therefore, physicians should become familiar with the essential tasks of the agents to determine if they are ready to carry out their activities [11].

Czaja-Miturai, et al. verified that when examining the TA in this type of personnel, it was elevated in $36 \%$, respiratory 
discomfort in $60 \%$, the BMI cholesterol, and low-density lipoproteins were high $(22.7+/-4.1,222.6+/-41.7 \mathrm{mg} / \mathrm{dl}$ and $142.7+/-39.7 \mathrm{mg} / \mathrm{dl}$, respectively). The general and occupational stress levels were: 34.914 .8 and $128.0+33.3$, respectively. In the group with the highest levels of stress, there were more circulatory problems $(81 \%)$, alcohol intake at least once a week (27\%), workdays in a 3-shift system (40.5\%) and work with overtime (44\%) [12].

To reduce the morbidity and mortality of cardiac diseases, Ramey, et al. [13] used a health promotion model planned with higher overweight and hypertension than in the general population, Diabetes mellitus being the greatest predictor of heart disease. Occupational health nursing personnel are trained to identify these health risks, designating appropriate interventions and convincing staff of healthy lifestyle changes.

De Sio, et al. [14] confirm that occupational exposure to urban stressors can increase systolic blood pressure in these workers during their work. The sources of stress for police officers have various origins: contact with the population, confrontation with lawbreakers, rotating work schedules, and the obligation to maintain high standards of service in different changing labor contexts [15].

Capozzella, et al. [16], in their study, when dividing the policemen by seniority, they found that the older ones are more exposed to stress and the risk of heart disease. Mallik, et al., Carried out a study similar to ours and they found a prevalence of hypertension, prehypertension, and normotension in 41.9, 42.9 and $15.2 \%$, respectively. A quarter of the agents under 40 years of age had hypertension although this increases with age and about $40 \%$ of hypertensive individuals knew their disease, $3 / 4$ parts of them received medical treatment although only one third had their blood pressure controlled TA $(<140 / 90 \mathrm{mmHg})$ [17].

In recent times it has been shown that the blood pressure figures that represent the risk of organ damage are that above $140 \mathrm{mmHg}$ for systolic pressure and $90 \mathrm{mmHg}$ for diastolic pressure when these remain in a sustained manner. Therefore, it is defined as arterial hypertension when on three different occasions greater than 140/90 $\mathrm{mmHg}$ are shown in the office or when ambulatory monitoring of blood pressure shows the presence of figures greater than those noted above. $50 \%$ of the registered intakes [18].

It is estimated that more than half of patients with hypertension have no control over their disease and to decrease the risk of heart attacks they must establish changes in style, life, disease control and reduce their complications $[19,20]$. Finally, one of the key risk factors for cardiovascular diseases is hypertension. It already affects one billion people in the world and can cause myocardial infarctions and cerebrovascular events. The researchers estimate that hypertension is the cause of nine million people dying each year. However, this risk does not necessarily have to be so high. Hypertension can be prevented. Prevention is much less expensive and much safer for patients than interventions such as myocardial revascularization surgery or dialysis, which are sometimes necessary when hypertension is not diagnosed and not treated.

\section{Conclusion}

The measures that must be taken into consideration to control this epidemic will be: health education, healthy diet, aerobic physical exercise, smoking cessation, reduction of alcohol and coffee consumption, including adrenergic stimulants, decreased salt intake in the diet and an optimal Body Mass Index. Or the control of Arterial Hypertension should intervene the patient, general physician or internist, nutritionist, nursing, psychology.

Given that stress, a few hours of rest and prolonged fasting are typical of one of the trades that have greater contact with the public predisposing to diseases (hypertension, obesity); It is recommended that work hours of 24 hours; be reduced to 8 hours or 12 hours thus giving the opportunity to rest at home for a period of time, and rejoin the workday optimizing their efficiency, effectiveness, and effectiveness to perform the activities of their trade.

\section{Acknowledgment}

To the students of the nursing degree of the Itzamna University Institute for the support in the taking of the blood pressure.

\section{References}

1. Organización Mundial de la Salud. Información General sobre Hipertensión Arterial en el Mundo. Día Mundial de la Salud. 2013; 1-40.

2. Organización Panamericana de la Salud. Prevención, Detección Evaluación y Tratamiento de la Hipertensión Arterial. OPS. 2008; 1-22.

3. Secretaria de Salud. Guide to Clinical Diagnostic Practice and Treatment of Arterial Hypertension in the First Level of Care. Quick Reference Guide. Catálogo Maestro de Guías de Práctica Clínica: IMSS-076-08. 2014; 3-21.

4. Instituto Mexicano del Seguro Social. Diagnosis and Treatment of Arterial Hypertension in the First Level of Care. Evidence and Recommendations. Catálogo Maestro de Guías de Práctica Clínica: IMSS-076-08. 2014; 1-77.

5. Ezekwesili CN, Ononamadu CJ, Onyeukwu OF, Mefoh NC Epidemiological survey of hypertension in Anambra State, Nigeria. Nig J Clin Pract. 2016; 19: 659-667.

PubMed: https://www.ncbi.nlm.nih.gov/pubmed/27538557

6. Díez J, Lahera V. Hipertensión arterial (I). Aspectos fisiopatológicos. Clin Invest Arterioscl. 2001; 13: 80-84.

7. Ganesh KS, Naresh AG, Bammigatti C. Prevalence and Risk Factors of Hypertension Among Male Police Personnel in Urban Puducherry, India. Kathmandu Univ Med J (KUMJ). 2014; 12: 242-246.

PubMed: https://www.ncbi.nlm.nih.gov/pubmed/26333577

8. Ramakrishnan J, Majgi SM, Premarajan KC, Lakshminarayanan S, Thangaraj $S$, et al. High prevalence of cardiovascular risk factors among policemen in Puducherry, South India. J Cardiovasc Dis Res. 2013; 4: 112-115.

PubMed: https://www.ncbi.nlm.nih.gov/pubmed/24027367 
9. Campos-Nonato I, Hernández-Barrera L, Rojas-Martínez R, Pedroza A, Medina-García C, Barquera-Cervera S. Hipertensión arterial: prevalencia, diagnóstico oportuno, control y tendencias en adultos mexicanos. Sal Púb Méx. 2013; 55: S144-S150.

10. Shiozaki M, Miyai N, Morioka I, Utsumi M, Koike H, et al. Assessment of the risk of ischemic heart disease and its relevant factors among Japanese police officers. Sangyo Eiseigaku Zasshi. 2013; 55: 115-124. PubMed: https://www.ncbi.nlm.nih.gov/pubmed/23676527

11. Zimmerman FH. Cardiovascular disease and risk factors in law enforcement personnel: a comprehensive review. Cardiol Rev. 2012; 20: $159-166$

PubMed: https://www.ncbi.nlm.nih.gov/pubmed/22314143

12. Czaja-Miturai I, Merecz-Kot D, Szymczak W, Bortkiewicz A Cardiovascular risk factors and life and occupational stress among policemen. Med Pr. 2013; 64: 335-348.

PubMed: https://www.ncbi.nlm.nih.gov/pubmed/24261247

13. Ramey SL, Downing NR, Knoblauch A. Developing strategic interventions to reduce cardiovascular disease risk among law enforcement officers: the art and science of data triangulation. AAOHN J. 2008; 56: 54-62. PubMed: https://www.ncbi.nlm.nih.gov/pubmed/18306648

14. De Sio S, Caciari T, Rosati MV, Casale T, Di Giorgio V, et al. Dynamic blood pressure in workers exposed to urban stressors. Ann Ig. 2013; 25 511-517. PubMed: https://www.ncbi.nlm.nih.gov/pubmed/24284537
15. Tomei F, Rosati MV, Baccolo TP, Cherubini E, Ciarroca M, et al. Ambulatory (24 hours) blood pressure monitoring in police officer. $J$ Occup Health. 2004; 46: 235-243.

PubMed: https://www.ncbi.nlm.nih.gov/pubmed/15215668

16. Capozzella A, Sancini A, De Sio S, Samperi I, Scala B, et al. Arterial pressure in workers exposed to urban stressors. $\mathrm{G}$ Ital Med Lav Ergon. 2015; 37: 20-25

PubMed: https://www.ncbi.nlm.nih.gov/pubmed/26193737

17. García A, Valero R. Normas, Consejos y Clasificación Sobre Hipertensión Arterial. Enferm Glob. 2009; 15: 1-14.

18. Mallik D, Mukhopadhyay DK, Kumar P, Sinhababu A. Hypertension, Prehypertension and Normotension among Police Personnel in a District of West Bengal, India. J Assoc Physicians India. 2014; 62: 12-16. PubMed: https://www.ncbi.nlm.nih.gov/pubmed/26281474

19. Valero R, García-Soriano A. Normas, consejos y clasificaciones sobre hipertensión arterial. Enfermería Global. 2008; 15: 1-14.

20. Valenzuela-Flores AA, Solórzano-Santos F, Valenzuela-Flores AG, Durán-Arenas LG, Ponce de León-Rosales S, et al. Key recommendations of the clinical guidelines of arterial hypertension in primary care. Rev Med Inst Mex Seguro Soc. 2016; 54: 249-260.

PubMed: https://www.ncbi.nlm.nih.gov/pubmed/26960054 